\title{
How well can we identify pseudobulges?
}

\author{
Alister Graham \\ Swinburne University of Technology Australia, email: agraham@astro.swin.edu.au
}

\begin{abstract}
Since the discovery of rotating galaxy bulges (e.g. Pease 1918; Babcock 1938, 1939), especially in the 1970s (e.g. Rubin, Ford \& Kumar 1973; Pellet 1976; Bertola \& Capaccioli 1977; Peterson 1978; Mebold et al. 1979; Kormendy \& Illingworth 1979), coupled with early computer simulations of disks which formed rotating, exponential-like "pseudobulges" (e.g. Bardeen 1975; Hohl 1975, and references therein), a number of often over-looked problems pertaining to the identification of real "pseudobulges" have arisen. Drawing on my recent review article of disk galaxy structure and modern scaling laws (Graham 2012), some of these important issues are presented. Topics include: classical spheroids with exponential light distributions; curved but continuous scaling relations involving the 'effective' structural parameters; the old age of most bulge stars (e.g. Thomas \& Davies 2006; MacArthur et al. 2009); that most disk galaxies have bulge-to-disk flux ratios $<1 / 3$ (Graham \& Worley 2008); rotation in simulated merger remnants (e.g. Bekki 2010; Keselman \& Nusser 2012) plus many other frustrating yet interesting reasons why rotation may not be a definitive signature of bulges built via secular processes (e.g. Babusiaux et al. 2010; Williams et al. 2010, Qu et al. 2011; Saha et al. 2012)
\end{abstract}

\section{References}

Babcock, H. W. 1938, PASP, 50, 174

Babcock, H. W. 1939, Lick Observatory Bulletin, 19, 41

Babusiaux, C., et al. 2010, A\&A, 519, A77

Bekki, K. 2010, MNRAS, 401, L58

Bardeen, J. M. 1975, IAU Symp., 69, 297

Bertola, F. \& Capaccioli, M. 1977, ApJ, 211, 697

Graham A. W., Worley, C. C. 2008, MNRAS, 388, 1708

Graham, A. W. 2012, in Planets, Stars, and Stellar Systems, Vol. 6, Extragalactic Astronomy and Cosmology, ed. W. C. Keel (New York: Springer-Verlag), in press (arXiv:1108.0997)

Hohl, F. 1975, IAU Symp., 69, 349

Keselman, J. A. \& Nusser, A. 2012, MNRAS, 424, 1232

Kormendy, J. \& Illingworth, G. 1979, Photometry, Kinematics and Dynamics of Galaxies, 195

MacArthur, L. A., González, J. J., \& Courteau, S. 2009, MNRAS, 395, 28

Mebold U., Goss W. M., Siegman B., van Woerden H., \& Hawarden T. G. 1979, A\&\&A, 74, 100

Pease, F. G. 1918, Proc. Nat. Acad. Sci., 4, 21

Pellet, A. 1976, A\&A , 50, 421

Peterson, C. J. 1978, ApJ, 221, 80

Qu, Y., Di Matteo, P., Lehnert, M. D., \& van Driel, W. 2011, A\&A, 530, A10

Rubin, V. C., Ford, W. K., Krishna, Kumar C. 1973, ApJ, 181, 61

Saha, K., Martinez-Valpuesta, I., \& Gerhard, O. 2012, MNRAS, 421, 333

Thomas, D. \& Davies R. L. 2006, MNRAS, 366, 510

Williams, M. J., et al. 2010, MNRAS, 414, 2163 